\title{
Aprimorando a Interação do Sistema de Ajuda de um Software Previdenciário
}

\author{
Douglas H. Pozzolo', Cristiano Maciel'1, Raphael de S. R. Gomes ${ }^{1}$ \\ ${ }^{1}$ Instituto de Computação - Universidade Federal de Mato Grosso (UFMT) - Cuiabá, \\ Mato Grosso - Brasil \\ douglas.pozzolo@gmail.com, cmaciel@ufmt.br, raphael@ic.ufmt.br
}

Abstract. In this research, Human-Computer Interaction methods and techniques are used in a social security software under development - WebRecad, specifically on its help system. Based on the results of usability tests and user experience evaluation methods, improvements to the software are proposed. These strategies also allow the company to take ownership of them in order to improve the development of their systems.

Resumo. Neste estudo são aplicados métodos e técnicas de Interação Humano-Computador em um software previdenciário em desenvolvimento - o WebRecad, mais especificamente no que se diz respeito ao seu sistema de ajuda. Com base nos resultados das avaliações de usabilidade e user experience, são propostas melhorias ao software. Tais estratégias permitem a empresa se apropriar delas para o desenvolvimento de seus sistemas.

\section{Introdução}

A interface de um sistema mal projetado pode causar grandes dificuldades, e até mesmo causar graves danos na realização de trabalhos de uma organização, e ainda assim, poucas organizações consideram este fato quando planejam suas aplicações (SHNEIDERMAN, 1997). Na área previdenciária, alguns softwares têm sido desenvolvidos. Entre eles, o WebRecad, um sistema de gerenciamento de segurados de um sistema previdenciário. Em parceria com sua empresa de desenvolvimento, a Webtech - Softwares e Serviços, percebeu-se que o mesmo, em sua fase de construção, não tem passado por um processo de revisão de usabilidade até o início do projeto deste artigo. Em uma primeira avaliação, percebe-se que a ajuda do usuário era falha. Face a esta problemática, questionou-se: como avaliações de usabilidade e de user experience (UX) podem agregar valor ao sistema de ajuda WebRecad, minimizando problemas com o uso dele? Evitar que o software falhe em quesitos como usabilidade, principalmente no que diz respeito à ajuda do sistema, tende a aumentar a qualidade de seu uso, é o que motiva o desenvolvimento deste trabalho.

Neste sentido, este artigo visa a utilização de métodos e técnicas da interação humano-computador (IHC) no contexto prático da produção do WebRecad, em seu sistema de ajuda. Para isto, o software foi sujeito às técnicas de avaliação: avaliação heurística, checklist, teste de usabilidade e user experience.

\section{Referencial Teórico}

No que se refere à experiência de utilização de um sistema, Nielsen (2007) conceitua usabilidade como elemento que garante a facilidade ao uso de algo. Assim, a avaliação 
de interface é imprescindível aos sistemas à medida que o desenvolvimento das interfaces dispõe de grandes desafios em relação às metodologias de projeto, que são vitais para ajudar cada ciclo de um sistema (BERTINI et al., 2009).

Antes de lançar um software para uso, é necessário identificar se o mesmo apoia de forma adequada seus usuários nas suas tarefas e em seu ambiente de uso. Seguir à risca métodos e princípios de projeto de interfaces não são suficientes para garantir uma alta qualidade de uso de um software. Desta forma, não somente testes de funcionalidade serão necessários para verificar a robustez da implementação, mas também a avaliação da interface será necessária para analisar a qualidade de uso do software (PRATES, BARBOSA, 2003). Exploraremos a seguir os conceitos das técnicas utilizadas neste artigo: a avaliação heurística, checklist, teste de usabilidade e avaliação de user experience.

A avaliação heurística é um método analítico de inspeção de interfaces criado por Jacob Nielsen e Molich (1993), que tem por objetivo identificar problemas de usabilidade baseado em conjunto de heurísticas (diretrizes) e até mesmo na experiência dos próprios avaliadores (NIELSEN, 1994). Este método é gerado das melhores práticas definidas por profissionais especialistas em IHC ao longo de anos trabalhados na área (PRATES; BARBOSA, 2003). Segundo Prates e Barbosa (2003), para cada heurística violada, o especialista deve definir a localização do problema e a sua gravidade (calculada com fatores como frequência, impacto na aplicação e persistência). Como produto final da avaliação, o especialista redige um relatório consolidado.

Por sua vez, a checklist é uma ferramenta de inspeção de usabilidade que tem poder de diagnosticar rapidamente problemas gerais e repetitivos das interfaces e pode ser usada com muita eficiência, não somente por profissionais especialistas em usabilidade, mas também programadores e analistas, por exemplo (JEFFRIES et al., 1991). Diferentemente da avaliação heurística, é a qualidade da lista de verificação (checklist), que determina as possibilidades da avaliação. Quanto melhor elaborada a checklist, maior a produção de resultados uniformes e abrangentes na definição dos problemas de usabilidade. Elas reduzem a subjetividade em relação ao processo de avaliação e são de rápida aplicação (CYBIS, 2003).

Por exemplificação, este trabalho faz uso de uma checklist, fruto de estudos contextualizados em softwares governamentais brasileiros criada por Maciel, Nogueira e Garcia (2005), denominada g-Quality. A checklist mapeia itens baseados nas heurísticas de Nielsen (1994) e no e-Ping (2004) - sigla de "Padrão de Interoperabilidade do Governo Eletrônico" - além de permitir avaliar acessibilidade, interoperabilidade, segurança e privacidade, veracidade da informação, agilidade do serviço e transparência de um sítio. Cabe ressaltar que o g-Quality tem visibilidade internacional (GARCIA et al., 2005) e, em alguns estudos, recortes dele tem sido utilizado e até atualizados, como em Silva, Maciel e Silva Junior (2020), neste caso, no que se refere à interoperabilidade.

O teste de usabilidade tem como foco a avaliação da qualidade da interação do usuário com o sistema, objetivando a métrica dos impactos sobre esta e a identificação dos aspectos da interface que geram desconforto ao usuário (CYBIS, 2017). De acordo com Santa Rosa (2008), os avaliadores produzem um roteiro com as tarefas que os participantes da pesquisa deverão realizar. O teste é realizado com a observação desta 
interação em um meio real ou sob condições controladas. Os avaliadores recolhem dados dos problemas encontrados através da interação dos usuários com a interface e verificam, posteriormente, se ela suporta o ambiente e as tarefas que o usuário executará (MATIAS, 1995). Procura-se quantificar, por meio deste teste, o desempenho do usuário. Dentre os exemplos de medidas no teste de usabilidade, é possível listar o tempo gasto para realizar uma tarefa, a quantidade de erros cometidos e a porcentagem dos usuários que conseguem se recuperar de um erro (PRATES, BARBOSA, 2003).

Além de usabilidade, deve-se levar em consideração que a atividade de um usuário num sistema tem relação direta na predisposição dele a utilizar um software, e a partir disso, se torna necessário que o software esteja em conformidade com a personalidade, emoção, humor, objetivos e preferência de seus usuários, e além disso, seu contexto físico, social e virtual onde irão ocorrer a interação com a interface (MCCARTHY; WRIGHT, 2007). Diversas técnicas foram criadas para compreender o estado emocional do usuário sobre cada detalhe do produto, para que a equipe de desenvolvimento seja capaz de tomar ações que causam melhorias na UX. Dentre elas, destaca-se os Emocards. Esta é uma técnica de avaliação não-verbal que consiste em mensurar respostas emocionais por meio de expressões faciais, permitindo assim validar as emoções expressadas independentemente da cultura do usuário (DESMET; OVERBEEKE; TAX, 2001). Ele consiste de 16 cartas com ilustrações faciais, sendo 8 masculinas e 8 femininas, representando possíveis emoções que contemplam dimensões de prazer e estimulação (SILVA; KRONBAUER, 2018).

\section{Metodologia}

Neste tópico serão descritos os métodos e técnicas utilizados juntamente com motivo das escolhas dos mesmos, além da a especificação da ferramenta WebRecad. Serão apresentados fluxos do processo realizado na produção do estudo e, em sequência, serão explicados em maiores detalhes.

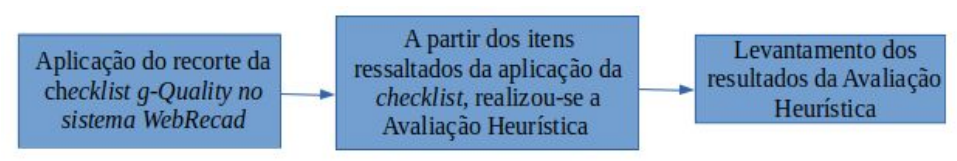

Figura 1 - Fluxo da criação dos resultados da Avaliação Heurística

\subsection{WebRecad}

O WebRecad é um sistema web em produção pela empresa Webtech - Softwares e Serviços designado ao recenseamento previdenciário, isto é, responsável por convocar e agendar segurados de um regime próprio de previdência social (RPPS) de uma determinada entidade governamental, para atualização de seus dados cadastrais e documentação comprobatória a fim de manter seus dados consistentes e seus benefícios validados dentro das regras da previdência social.

O fluxo principal do WebRecad pode ser dividido em cinco etapas: 1) cadastro de parâmetros, onde são definidos todos os parâmetros necessários para o recadastramento, por exemplo, os tipos de documentos que podem ser apresentados; 2) cadastro de projetos de recadastramento, em que se identifica as singularidades de um projeto de recadastramento, como quais documentos serão tidos como obrigatórios, se haverá coleta de biometria e fotos 3) convocação e agendamento, no qual, a partir dos projetos 
de recadastramento, é possível realizar a convocação dos segurados, filtrando os segurados de forma personalizada, por mês de nascimento, por exemplo. 4) a triagem, que trata-se da verificação dos documentos apresentados pelo segurado para averiguar se está com toda a documentação obrigatória para realizar a etapa de recadastramento; e 5) o recadastramento, a etapa fundamental de atualização dos dados cadastrais e apresentação de seus documentos comprobatórios.

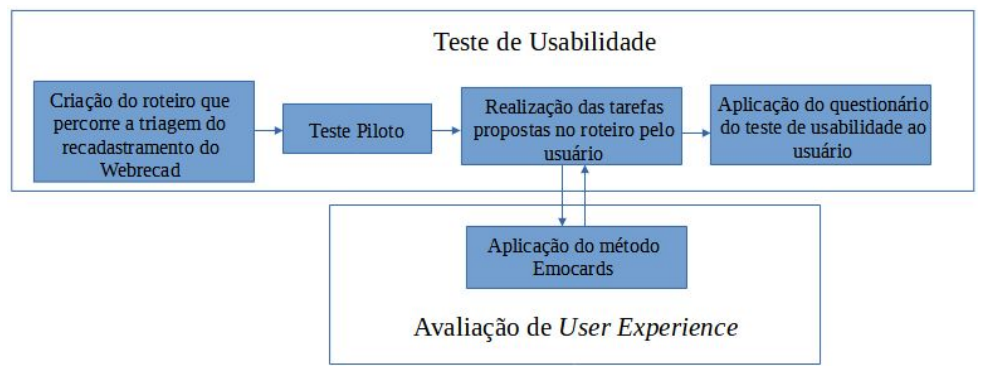

Figura 2 - Aplicação do teste de usabilidade e avaliação de user experience

O WebRecad possui, também, uma Central de Ajuda para o usuário, lá encontram-se dois manuais: um no formato PDF possuindo 49 páginas para operadores e 82 páginas para administradores, e outro em formato HTML (Linguagem de Marcação de HiperTexto) que é dividido em tópicos para cada módulo do sistema. No manual em HTML, existe um sistema de busca no qual é possível fazer uma busca pelo nome dos tópicos desejados.

\section{2 g-Quality}

No intuito de avaliar os métodos de ajuda na interface do WebRecad, utilizou-se um recorte da checklist utilizada no estudo do g-Quality (MACIEL; NOGUEIRA; GARCIA, 2005) no que diz respeito à parte que trata da heurística de Nielsen "Ajuda e Documentação". Como este foi criado para análise de interfaces de sítios governamentais, se enquadra como uma potencial ferramenta para a avaliação do WebRecad. Os itens da checklist identificados como problema de usabilidade serão utilizados como base para a avaliação heurística no passo posterior. A checklist adaptada foi aplicada em dezembro de 2019 e preenchida conforme proposto no método.

\subsection{Avaliação Heurística}

A partir de cada problema de interface identificado com a checklist, foi realizada uma avaliação heurística sobre o quesito. Com no artigo "Avaliação Heurística de Sítios na Web" de Maciel et al. (2004) e no formulário fornecido neste, foi possível classificar, atribuir um grau de severidade ao problema, identificar a causa e os efeitos trazidos por ele sobre o usuário e a tarefa, e propor uma possível solução.

\subsection{Teste de Usabilidade}

Como método empírico de avaliação de interface, utilizou-se o teste de usabilidade. Para tal, foi criado um roteiro de tarefas as quais deveriam ser realizadas pelos usuários (Figura 2). Este teste teve como objetivo induzir o usuário, por meio de tarefas dentro do WebRecad, a utilizar a central de ajuda do sistema, e assim, criar um feedback sobre a qualidade e eficiência do sistema denominado "Central de Ajuda". O teste foi 
realizado na sede da Webtech por 5 participantes com perfil de operador WebRecad, com os critérios de que tivessem acima de 18 anos de idade, noções de informática básica e que não tivessem contato prévio com o sistema. Todo o procedimento foi gravado com o consentimento do usuário, tanto a tela da interface, quanto o rosto do participante durante a realização da avaliação. Realizou-se, anteriormente, um teste piloto com um avaliador para que se testasse a qualidade do roteiro criado e se seus aspectos - objetos de avaliação - fossem postos à prova. Os dados recolhidos neste teste não foram contabilizados.
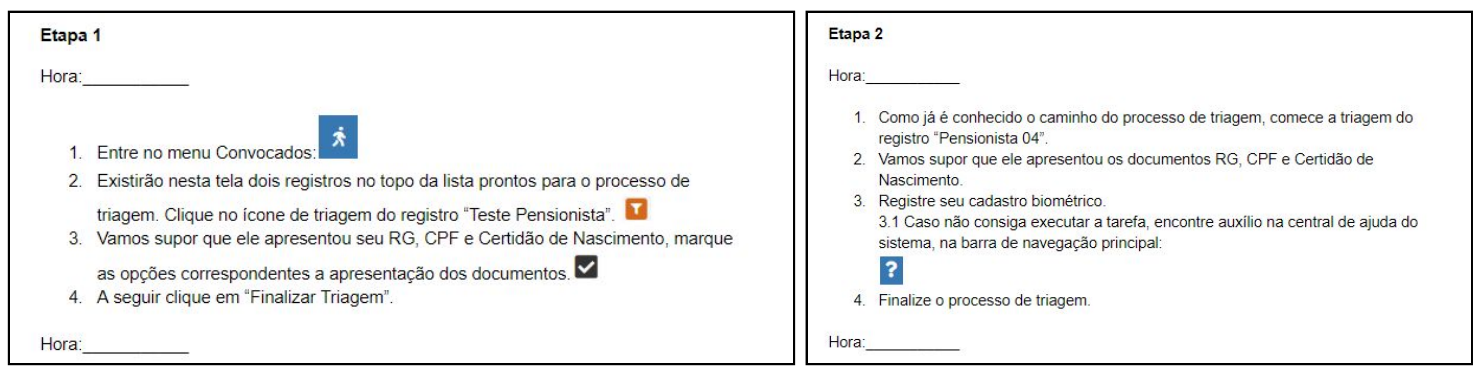

Figura 3 - Etapas do roteiro do Teste de Usabilidade

O roteiro foi escrito para a realização de dois processos de triagem no WebRecad. Com dois registros de segurados já preparados previamente, foi pedido aos usuários que o realizassem de acordo com as instruções do roteiro, que possui duas etapas (Figura 3): a primeira, demonstrando passo a passo de como realizar um processo de triagem simples, sem cadastramento de biometria; na segunda etapa, já com menos detalhamento sobre o procedimento, pediu-se que o usuário realizasse o processo de triagem para outro registro, mas desta vez com cadastro biométrico, uma tarefa mais complexa. No intuito de fazer com que o usuário buscasse auxílio na central de ajuda do sistema existia uma observação abaixo da tarefa: "Caso não consiga executar a tarefa, encontre auxílio na central de ajuda do sistema" com informações de como acessá-lo, indicando a existência de uma central de ajuda, onde se localiza o manual de usuário. Ambas etapas possuem um campo para registro das horas a fim de medir o tempo que o usuário levou para a realização das tarefas. Ao finalizar o teste, o usuário foi submetido a um questionário com perguntas-chave sobre a facilidade de uso do sistema e a necessidade de auxílio na realização das atividades.

\subsection{Avaliação de UX}

Conforme ilustrado na Figura 2, simultaneamente com o teste de usabilidade, foi realizada uma avaliação de user experience. Para tal, foi entregue uma página com Emocards (Desmet; Overbeeke; Tax, 2001) (Figura 4), sendo que, ao realizar cada uma das etapas da tarefa, o usuário foi orientado a selecionar um card que mais se aproxima da emoção sentida ao realizá-la. A técnica foi escolhida para que a emoção do usuário sobre cada uma das etapas seja, de forma não-verbal, reconhecida e analisada a reconhecer o grau de satisfação do usuário ao interagir com a interface em virtude das prováveis dificuldades encontradas. Foi solicitado ao usuário anotar o número (de 1 a 8) que representa a emoção sentida em relação a cada um dos passos do roteiro. Utilizaram-se os Emocards originais, em conjunto com as orientações, sendo que o aplicador do teste os traduziu e esclareceu dúvidas sobre o método aos participantes. 


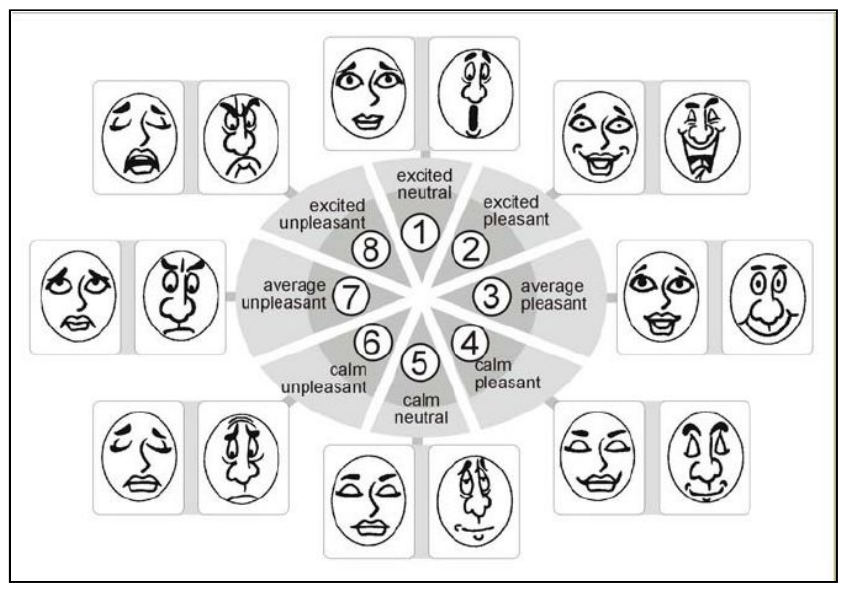

Figura 4 - Emocards de Desmet, Overbeeke \& Tax (2001).

\section{Resultados}

A seguir, serão apresentados os resultados das aplicações das metodologias.

\section{1 g-Quality}

A aplicação do recorte de "Ajuda e documentação", da checklist do método g-Quality no sistema do WebRecad, permitiu verificar os itens conforme elencado na Tabela 1. A primeira coluna identifica qual item da checklist está sendo verificado, em "Opção" foram escolhidos números de 0 a 2 baseados nos critérios: 0 para "nunca/opção não testável"; 1 para "às vezes" e 2 para "sempre", de acordo com o proposto no método.

Tabela 1. Verificação da Checklist - Ajuda e Documentação

\begin{tabular}{|l|c|}
\hline Item da checklist & Opção \\
\hline 1. Fornece recurso de ajuda (help) integrado com as páginas do sítio. & 2 \\
\hline 2. Não disponibiliza ajudas muito extensas. & 0 \\
\hline 3. Disponibiliza de ajudas contextualizadas. & 1 \\
\hline 4. A informação está organizada de forma organizada, com índice de separação entre as áreas. & 1 \\
\hline 5. Partes do sistema não têm acesso a ajuda. & 2 \\
\hline 6. Falta ajuda para determinados contextos ou ações. & 2 \\
\hline 7. Falta mecanismo de busca na ajuda. & 0 \\
\hline 8. Disponibiliza uma FAQ - Frequently Asked Questions (Perguntas Mais Frequentes). & 0 \\
\hline 9. Utiliza recursos de atendimento pessoal de forma online e em tempo real. & 0 \\
\hline
\end{tabular}

\subsection{Avaliação Heurística}

A avaliação heurística foi realizada com base no proposto na literatura e verificando cada item elencado como problema de usabilidade na checklist previamente apresentada. Percebe-se que o WebRecad possui apenas o manual do usuário integrado no sistema como fonte de auxílio e por ser um material muito extenso, pode causar muita hesitação no seu uso por parte dos usuários. Ficar revirando páginas e páginas de texto pode ser um tanto enfadonho, no entanto, o sistema acerta ao possuir um sistema de busca em seu manual HTML, sendo possível procurar palavras-chave dentre os 
títulos dos tópicos. Sair do contexto de uso para acessar a central de ajuda também é outro fator que pode causar retrabalho ao usuário, além desconforto e aborrecimento.

Com o preenchimento dos formulários, observa-se que a solução indicada para quase todos os problemas de usabilidade, no que se refere à ajuda do sistema, convergem para a adição de ajuda contextualizada nas páginas do sistema. É sugerido o uso de pequenos ícones próximos a campos e ações que possam gerar dúvidas aos usuários, que ao clicá-los, abre-se um pequeno balão explicativo sobre para que serve tal campo, ou o que faz tal ação. É sugerido também, para maximizar o uso do manual dentro do sistema, links dentro destes balões que, abrem uma segunda janela do navegador exatamente na página em que é explicado seu uso no manual. Outra sugestão é a existência de vídeos tutoriais para as ações que geram as dúvidas mais frequentes. É interessante destacar, logo ao entrar na central de ajuda, uma espécie de FAQ (perguntas mais frequentes, traduzido de Frequently Asked Questions) com a solução ou a descrição dos campos mais complexos, a apresentação do significado das siglas utilizadas, ou ações que mais geram dúvida nos usuários enquanto utilizam o sistema. A pesquisa de da Silva et al. (2013) enfatiza o uso de ajudas contextualizadas em sistemas de governo eletrônico.

\subsection{Teste de Usabilidade}

O teste de usabilidade foi aplicado na própria sede da Webtech com a participação de 5 usuários que se encaixam no perfil de operador WebRecad. A análise do resultado será dividida em 3 seções: primeira etapa do roteiro, segunda etapa do roteiro e o questionário. A tarefa a ser realizada pelo participante foi de realizar a triagem do registro de um segurado previamente agendado para o momento do teste. $\mathrm{O}$ procedimento a ser executado pelo usuário consistia em entrar na página dos registros, encontrar o registro descrito no roteiro e sinalizar de que este apresentou todos os documentos requeridos e a seguir, encerrar o processo de triagem.

A maior dificuldade encontrada pelos usuários na etapa 1 da tarefa (Figura 3) foi a localização do menu, uma vez que estava disposto em ícone, sem legenda visível, sendo necessário passar o ponteiro do mouse sobre ele para encontrar a descrição “Convocados". É questionável o símbolo do ícone, que mesmo estando explícito no roteiro, pode ser apontado como item de confusão aos usuários, sendo necessário um estudo sobre um ícone mais adequado. Na segunda etapa, a tarefa a ser realizada pelo participante é de realizar a triagem de um segundo segurado, porém desta vez, realizando um cadastro biométrico, utilizando os próprios dedos para simular os do segurado que está passando pela triagem. Mesmo sem possuir uma legenda visível na área de cadastramento de biometria, contendo apenas um ícone de leitura biométrica, a maioria (80\%) dos usuários reconheceram rapidamente o local para realizar o procedimento. Ainda que $100 \%$ dos usuários tiveram dúvidas em relação ao cadastro biométrico, nenhum deles recorreu ao auxílio da Central de Ajuda. Existiu total hesitação por parte dos usuários de sair do contexto atual (janela de cadastro de biometria) para buscar auxílio no sistema, o fato é realçado ao observar o usuário passando o mouse por cima do ícone em momento de dificuldade, mas ainda assim preferir clicar nos botões dispostos para testar a resposta da interface. Com a aplicação do questionário após a utilização do sistema pelos participantes, observa-se que a central de ajuda precisa ser reestruturada no WebRecad. 
Todos os usuários alegaram encontrar dificuldades ao usar o sistema, porém houve grande hesitação ao entrar numa página nova para encontrar ajuda. A preferência por tentativa e erro deve ser evitada ao máximo pela interface, uma vez que pode gerar danos à integridade dos dados e diminuir a satisfação do usuário com a interface. Há entre eles uma grande preferência $(80 \%)$ por vídeos tutoriais a longos manuais. Ao apresentar uma nova ideia de ajuda no sistema, $100 \%$ disseram que recorreriam a uma forma de ajuda na própria janela que está realizando as atividades.

\subsection{Avaliação de User Experience}

A avaliação de UX foi realizada em conjunto ao teste de usabilidade, assim que o usuário realizasse um passo na lista de tarefas, ele foi orientado a escolher um card de emoção, da técnica Emocards (DESMET; OVERBEEKE; TAX, 2001), correspondente à experiência do contato com a interface. Os emocards são numerados de 1 a 8 conforme a Figura 4. A tabela 2 demonstra o Emocard escolhido pelos usuários na realização dos passos do roteiro do teste de usabilidade (Figura 3).

Tabela 2. Resultados do Teste de UX

\begin{tabular}{|c|c|c|c|c|c|c|c|c|}
\hline & \multicolumn{5}{|c|}{ Etapa 1 } & \multicolumn{5}{c|}{ Etapa 2 } \\
\hline & Passo 1 & Passo 2 & Passo 3 & Passo 4 & Passo 1 & Passo 2 & Passo 3 & Passo 4 \\
\hline Usuário 1 & 6 & 4 & 4 & 4 & 7 & 7 & 8 & 2 \\
\hline Usuário 2 & 6 & 5 & 4 & 3 & 3 & 3 & 7 & 3 \\
\hline Usuário 3 & 7 & 3 & 6 & 1 & 3 & 3 & 5 & 3 \\
\hline Usuário 4 & 3 & 3 & 3 & 3 & 3 & 3 & 4 & 3 \\
\hline Usuário 5 & 5 & 7 & 5 & 5 & 5 & 5 & 7 & 5 \\
\hline
\end{tabular}

Analisando a numeração dos Emocards, as emoções consideradas positivas se referem às de número 1 a 4,5 é uma emoção completamente neutra, enquanto as emoções de 6 a 8 são emoções negativas que aumentam de intensidade à medida em que se aproximam do card 8. Na etapa 1 observa-se que o passo 1 gerou um grande número de reações negativas (60\%), no passo 2 ocorrem duas reações negativas, apenas uma delas com grande intensidade, no passo 3 ocorre grande quantidade de reações neutras e duas consideradas pouco desagradáveis, na etapa 4 ocorre a maior parte de reações positivas. Na segunda etapa do teste, os passos 1 e 2 possuem $60 \%$ de reações positivas, $20 \%$ muito desagradável e $20 \%$ levemente desagradável. O passo 3 é o que gerou maior quantidade de reações negativas com maior intensidade de todo o teste e em seguida, o quarto passo trouxe emoções positivas $(80 \%)$ em sua maioria.

Ao comparar os resultados do teste de usabilidade com o teste de user experience podemos notar que o pico de reações negativas gerada pelo passo 1 da etapa 1 ocorre pela dificuldade do usuário de encontrar o menu, da forma que ele disposto na interface. Já o pico de reações negativas gerado pelo passo 3 da etapa 2 ocorre pela dificuldade enfrentada pelo usuário de realizar a tarefa, nota-se que nesta etapa, mais do que todas as outras, o usuário necessita de ajuda. Com a análise das imagens gravadas, a etapa de começar o cadastro biométrico é facilmente encontrada e o incômodo da dificuldade apresentada nos dados tem início no momento em que o usuário interage com o botão "Capturar" e utiliza o leitor biométrico, e retorna no momento em que é necessário posicionar o dedo no leitor novamente para confirmação da digital. 


\section{Considerações Finais}

Todos os métodos utilizados para a avaliação de interface trouxeram informações primordiais para um estudo mais aprofundado sobre como melhorar a qualidade e a humanização da interação do usuário com a ajuda do sistema. Embora o teste de usabilidade ter sido mais custoso, em questão de tempo de preparo e aplicação, trouxe informações mais ricas sobre os problemas de usabilidade, encontrando até mesmo problemas fora do contexto de ajuda e documentação, como ícones inadequados e menus de navegação confusos.

A avaliação heurística, juntamente com a checklist, indicaram que o sistema de ajuda não teria alta probabilidade de eficiência, uma vez que o manual do usuário, que apesar de ser muito rico em detalhes, é muito extenso. Além de destacar que o redirecionamento do usuário a uma página nova, saindo do contexto do seu problema para buscar ajuda, causa hesitação dos usuários ao utilizar a ferramenta. O teste de usabilidade, quando aplicado com o método dos Emocards, não somente confirmou a hesitação dos usuários em utilizar a ferramenta de ajuda como também provou que nenhum sequer a utilizou.

De forma geral, todo o estudo aponta que o WebRecad precisa reformular seu método de ajuda, sugerindo, entre outras formas, a utilização de ajuda contextual além da possível implementação de vídeos tutoriais ou links que, além de abrirem uma nova janela (para não ocorrer o risco de sair do contexto do problema), revelam explicação mais detalhada da dúvida dentro do próprio manual do usuário já existente. Todos os resultados obtidos pela aplicação dos testes e conceitos de IHC na interface do WebRecad apontaram elementos que, ao serem trabalhados, trarão maior qualidade ao software final. Isso reitera a importância da aplicabilidade de métodos, técnicas e ferramentas de IHC dentro de corporações e projetos de software no que diz respeito a qualidade do uso e, por consequência, o produto final.

Os resultados gerados por esta pesquisa foram repassados para a própria empresa, para que se estude, juntamente com suas normas de criação e seus desenvolvedores, o melhor meio de aprimorar a ferramenta de ajuda e documentação dentro, não somente do WebRecad, mas de todos seus sistemas. Assim, ficou claro, no ambiente empresarial em que esse software é utilizado, que os aspectos humanos são fundamentais no desenvolvimento dos sistemas, e precisam sempre ser considerados, uma vez que podem afetar, inclusive, os aspectos comerciais da empresa.

\section{Referências}

Bertini, E., Catarci, T., Dix, A., Gabrielli, S., Kimani, S., \& Santucci, G. (2009). Appropriating heuristic evaluation for mobile computing. International Journal of Mobile Human Computer Interaction (IJMHCI), 1(1), 20-41.

Cybis, W. D. A. (2003). Engenharia de usabilidade: uma abordagem ergonômica. Florianópolis: Labiutil.

Cybis, W., Betiol, A. H., \& Faust, R. (2017). Ergonomia e usabilidade: conhecimentos, métodos e aplicações. Novatec editora. 
da Silva, Liziane A. et al. Designing help system for e-GOV websites: A Brazilian case study. Information Polity, v. 18, n. 3, p. 261-274, 2013.

Desmet, P., Overbeeke, K., \& Tax, S. (2001). Designing products with added emotional value: Development and appllcation of an approach for research through design. The design journal, 4(1), 32-47.

Garcia, A. C. B., Maciel, C., \& Pinto, F. B. (2005). A quality inspection method to evaluate e-government sites. In International Conference on Electronic Government (pp. 198-209). Springer, Berlin, Heidelberg.

Jeffries, R., Miller, J. R., Wharton, C., Uyeda, K. (1991). User interface evaluation in the real world: A comparison of four techniques. In proceedings of CHI'91. In Conference on Human Factors and Computing Systems, New Orleans, LA (pp. 119-124).

Maciel, C., Nogueira, J. L. T., Ciuffo, L. N., Garcia, A. C. B. Avaliação heurística de sítios na web. VII Escola de Informática da SBC-Centro-Oeste, 2004.

Maciel, C.; Nogueira, J.L.T.; Garcia, A.C.B. An x-ray of the brazilian e-gov web sites. In: IFIP Conference on Human-Computer Interaction. Springer, Berlin, Heidelberg, 2005. p. 1138-1141.

Maciel, C.; Nogueira, J.L.T.; Garcia, A.C.B. g-Quality: um método para avaliação da qualidade dos sítios de e-Gov. VIII Escola de Informática do SBC - Centro-Oeste, Cuiabá. SUCESU-MT. PAK Multimídia, Cuiabá, 2005.

McCarthy, J.; Wright, P. (2007) Technology as experience. The MIT Press.

Nielsen, J. (1994). Heuristic evaluation. In Usability inspection methods (pp. 25-62). John Wiley \& Sons, Inc..

Nielsen, J. (2007). Loranger, Hoa. Usabilidade na web: Projetando websites com qualidade. Rio de Janeiro: Elsevier.

Prates, R. O., Barbosa, S. D. J. (2003). Avaliação de interfaces de usuário-conceitos e métodos. In Jornada de Atualização em Informática do Congresso da Sociedade Brasileira de Computação, Capítulo (Vol. 6, p. 28).

Shneiderman, B. (1997). Designing the user interface: strategies for effective human-computer interaction. New York: Addison-Wesley, 1997.

Silva, B., Maciel, C; Silva Junior, D. Interoperability Inspection Method in Electronic Government based on e-PING Architecture. In XVI Simpósio Brasileiro de Sistemas de Informação, SBC, 2020. 